S. KATO

KODAI MATH. J.

18 (1995), $86-98$

\title{
CONSTRUCTION OF n-END CATENOIDS WITH PRESCRIBED FLUX
}

\author{
SHIN KATO
}

\section{Introduction}

Let $X: \hat{\boldsymbol{C}}-\left\{q_{1}, \cdots, q_{n}\right\} \rightarrow \boldsymbol{R}^{3}$ be an $n$-end catenoid, that is, a complete minimal surface of genus 0 having $n$ catenoid ends at $q_{i}$ 's, where $\widehat{\boldsymbol{C}}:=\boldsymbol{C} \cup\{\infty\}$. Let $G: \widehat{\boldsymbol{C}}-\left\{q_{1}, \cdots, q_{n}\right\} \rightarrow \boldsymbol{S}^{2}$ be its Gauss map which can be extended naturally on $\widehat{\boldsymbol{C}}$, and let $w\left(q_{i}\right)$ denote the weight of the end $q_{i}$, that is, the similitude ratio of the asymptotic catenoid of the end $q_{i}$ to the standard catenoid ( $g=-z, \eta=$ $-z^{-2} d z$ ). Remark that $w\left(q_{i}\right)$ takes negative value if the orientation of the end $q_{2}$ differs from that of the standard catenoid, and that $w\left(q_{i}\right)$ vanishes if the end $q_{2}$ is a flat end or is removed. The vector $w\left(q_{i}\right) G\left(q_{i}\right)$ is called the flux vector of the end $q_{\imath}$ and, it follows from the flux formula (cf. e. g. [2]) that $\sum_{\imath=1}^{n} w\left(q_{i}\right) G\left(q_{i}\right)$ $=0$. Now, conversely, we consider the following

PROBLEM. Given $n$ unit vectors $v_{1}, \cdots, v_{n}$ in $\boldsymbol{R}^{3}$ and $n$ non-zero real numbers $a_{1}, \cdots, a_{n}$ satisfying $\sum_{\imath=1}^{n} a_{i} v_{i}=0$, is there an $n$-end catenoid $X: \hat{\boldsymbol{C}}-\left\{q_{1}, \cdots, q_{n}\right\}$ $\rightarrow \boldsymbol{R}^{3}$ such that $G\left(q_{i}\right)=v_{\imath}$ and $w\left(q_{i}\right)=a_{\imath}$ ?

In this paper, we study the problem in the case when $q_{2}$ coincides with $\boldsymbol{\sigma}\left(v_{\imath}\right)$ for each $i$, where $\sigma: \boldsymbol{S}^{2} \rightarrow \widehat{\boldsymbol{C}}$ is the stereographic projection from the north pole. Our main result is stated as follows.

THEOREM. Let $v_{1}, \cdots, v_{n}$ be unit vectors in $\boldsymbol{R}^{3}$, and $a_{1}, \cdots, a_{n}$ non-zero real numbers satisfying $\sum_{\imath=1}^{n} a_{i} v_{\imath}=0$. Set $p_{\imath}:=\sigma\left(v_{\imath}\right)$ and

$$
F_{i}(z):=\frac{\bar{p}_{i} z+1}{z-p_{i}} .
$$

Suppose there are complex numbers $b_{1}, \cdots, b_{n}$ satisfying

$$
\begin{aligned}
& b_{i} \sum_{j \in N_{\imath}} b_{j}=a_{\imath} \quad \imath=1, \cdots, n, \\
& \sum_{j \in N_{\imath}} b_{j} F_{i}\left(p_{j}\right)=0 \quad i=1, \cdots, n
\end{aligned}
$$

and $\sum_{\imath=1}^{n} b_{i} \neq 0$, where $N_{\imath}:=\{j \in N \mid 1 \leqq j \leqq n, j \neq i\}$. Then there exists an $n$-end

Received September 27, 1993; revised April 1, 1994. 
catenoid $X: \widehat{\boldsymbol{C}}-\left\{p_{1}, \cdots, p_{n}\right\} \rightarrow \boldsymbol{R}^{3}$ such that $G\left(p_{\imath}\right)=v_{\imath}$ and $w\left(p_{\imath}\right)=a_{\imath}$.

In Section 2, we prove this theorem by giving explicit representation for the solution surface.

Except for the (2-end) catenoid, examples of $n$-end catenoids were first introduced by Jorge-Meeks [3]. These are the case with $p_{i}=\zeta_{n}^{2}$ and $a_{i} \equiv 1$, where $\zeta_{n}$ is a primitive root of the equation $z^{n}=1$. (Throughout this paper, we keep this notation.) Subsequently, Karcher [4] constructed some new 4-end catenoids, and Lopez [5] classified all of the 3-end catenoids. In each of these examples, $v_{1}, \cdots, v_{n}$ lies on the same great circle in $\boldsymbol{S}^{2}$.

Recently, Xu [9], Rossman [7] and Umehara-Yamada [8] constructed polyhedrally symmetric $n$-end catenoids and some less symmetric ones, in each of which $v_{1}, \cdots, v_{n}$ do not lie on the same great circle in $\boldsymbol{S}^{2}$. For this purpose, $\mathrm{Xu}$ used directly the Enneper-Weierstrass representation. On the other hand, Rossman employed the conjugate surface method and constructed also higher genus examples (see also Berglund-Rossman [1]). Umehara and Yamada constructed polyhedrally symmetric ones as limits of those corresponding CMC-c surfaces in $\boldsymbol{H}^{3}\left(-c^{2}\right)$.

Each example of $n$-end catenoids in Jorge-Meeks [3] and $\mathrm{Xu}$ [9] has the ends of the same weight, and it is easy to observe that they are all described by the following special case of our theorem.

COROLLARY. Let $v_{1}, \cdots, v_{n}$ be unit vectors satısfying $\sum_{i=1}^{n} v_{i}=0$, and $p_{\imath}, F_{2}$ and $N_{\imath}$ as in Theorem. If

$$
\sum_{\jmath \in N_{\imath}} F_{i}\left(p_{\jmath}\right)=0 \quad i=1, \cdots, n,
$$

then there exists an $n$-end catenoid $X: \widehat{\boldsymbol{C}}-\left\{p_{1}, \cdots, p_{n}\right\} \rightarrow \boldsymbol{R}^{3}$ such that $G\left(p_{\imath}\right)=v_{\imath}$ and $w\left(p_{\imath}\right) \equiv 1$.

Finally, we emphasize that almost all of the known examples can be constructed by our theorem. In Section 3, we discuss this and also give far more new examples of families of $n$-end catenoids having ends of 2,3 or 4 different weights.

The author would like to express sincere gratitude to Professors O. Kobayashi, S. Nayatani, W. Rossman, M. Umehara and K. Yamada for their constant encouragement and valuable conversations.

\section{Proof of Theorem}

In this section, we prove our main theorem. First, we recall the following famous and significant

ENNEPER-WEIERSTRASS REPRESENTATION. (cf. [6]) Let $\Sigma$ be a Riemann 
surface, $g$ a meromorphic function on $\Sigma$, and $\eta$ a holomorphic 1-form on $\Sigma$. Define a map $X: \Sigma \rightarrow \boldsymbol{R}^{3}$ by

$$
X(z)=\operatorname{Re} \int^{z}\left(1-g^{2}, \sqrt{-1}\left(1+g^{2}\right), 2 g\right) \eta .
$$

If

$$
\operatorname{Re} \int_{C}\left(1-g^{2}, \sqrt{-1}\left(1+g^{2}\right), 2 g\right) \eta=0
$$

for any closed curve $C$ on $\Sigma$, then $X$ is a conformal minimal branched immersion whose Gauss map is $\sigma^{-1} \circ g$. Moreover, the induced metric of $\Sigma$ is given by

$$
d s^{2}=\left(1+|g|^{2}\right)^{2}|\eta|^{2} .
$$

Proof of Theorem. First, we assume $p_{\imath} \neq \infty$ for any $\imath$. Set

$$
f(z):=\sum_{i=1}^{n} \frac{b_{i}}{z-p_{i}}, \quad \beta:=\sum_{i=1}^{n} b_{i}
$$

and

$$
g(z):=z-\frac{\beta}{f(z)}, \quad \eta:=-\{f(z)\}^{2} d z
$$

We will show that the surface $X: \widehat{\boldsymbol{C}}-\left\{q_{1}, \cdots, q_{n}\right\} \rightarrow \boldsymbol{R}^{3}$ represented by these data is an $n$-end catenoid we want to construct.

Let $\left(v_{\imath 1}, v_{i 2}, v_{i 3}\right)$ be the orthogonal coordinate of the vector $v_{i}$. Then, by using the assumptions (1.1) and (1.2), we have, for any $i$,

$$
\begin{aligned}
\operatorname{Res}_{z=p_{i}}\left\{-\left(1-g^{2}\right) f^{2}\right\} & =2 b_{i} \sum_{\jmath \in N_{\imath}} b_{j} \frac{p_{\imath} p_{j}-1}{p_{i}-p_{\jmath}} \\
& =-2\left\{b_{i}\left(\beta-b_{i}\right) \frac{p_{i}+\bar{p}_{\imath}}{\left|p_{\imath}\right|^{2}+1}+\frac{b_{i}\left(p_{i}^{2}-1\right)}{\left|p_{\imath}\right|^{2}+1} \sum_{\jmath \in N_{\imath}} b_{j} F_{\imath}\left(p_{j}\right)\right\} \\
& =-2 a_{i} v_{i 1} \in \boldsymbol{R}, \\
\operatorname{Res}_{z=p_{i}}\left\{-\sqrt{-1}\left(1+g^{2}\right) f^{2}\right\} & =\frac{2 b_{i}}{\sqrt{-1}} \sum_{\jmath \in N_{\imath}} b_{j} \frac{p_{\imath} p_{j}+1}{p_{i}-p_{\jmath}} \\
& =-\frac{2}{\sqrt{-1}}\left\{b_{i}\left(\beta-b_{i}\right) \frac{p_{i}-\bar{p}_{\imath}}{\left|p_{\imath}\right|^{2}+1}+\frac{b_{i}\left(p_{i}^{2}+1\right)}{\left|p_{\imath}\right|^{2}+1} \sum_{\jmath \in N_{\imath}} b_{\jmath} F_{\imath}\left(p_{\jmath}\right)\right\} \\
& =-2 a_{i} v_{i 2} \in \boldsymbol{R}, \\
\operatorname{Res}_{z=p_{i}}\left\{-2 g f^{2}\right\} & =-2 b_{i} \sum_{\jmath \in N_{\imath}} b_{j} \frac{p_{i}+p_{\jmath}}{p_{i}-p_{\jmath}} \\
& =-2\left\{b_{i}\left(\beta-b_{i}\right) \frac{\left|p_{\imath}\right|^{2}-1}{\left|p_{\imath}\right|^{2}+1}-\frac{2 b_{i} p_{\imath}}{\left|p_{\imath}\right|^{2}+1} \sum_{j \in N_{\imath}} b_{j} F_{i}\left(p_{j}\right)\right\} \\
& =-2 a_{i} v_{i 3} \in \boldsymbol{R} .
\end{aligned}
$$


Hence the condition (2.1) holds, and the surface $X$ is well-defined. Moreover, since $\beta \neq 0$, the induced metric

$$
d s^{2}=\left(|f|^{2}+|z f-\beta|^{2}\right)^{2}|d z|^{2}
$$

is non-degenerate. By simple calculation, we get the following expansions around $p_{i}$.

$$
\begin{array}{r}
g(z)=p_{i}-\frac{\beta-b_{i}}{b_{i}}\left(z-p_{\imath}\right)+O\left(\left(z-p_{\imath}\right)^{2}\right), \\
\eta=\left\{-\frac{b_{i}^{2}}{\left(z-p_{\imath}\right)^{2}}+O\left(\frac{1}{z-p_{i}}\right)\right\} d z .
\end{array}
$$

Therefore, for any $i$, the surface $X$ has a catenoid end at $p_{\imath}$ such that $G\left(p_{\imath}\right)$ $=\sigma^{-1} \circ g\left(p_{i}\right)=v_{i}$ and

$$
w\left(p_{\imath}\right)=-\frac{\beta-b_{i}}{b_{i}}\left(-b_{i}^{2}\right)=b_{i}\left(\beta-b_{i}\right)=a_{\imath} .
$$

On the other hand, it is easy to see that, even if $p_{\imath}=\infty$ for some $\imath$, the assertion of Theorem and the data (2.2) are valid in the sense that

$$
\begin{aligned}
& F_{i}\left(p_{j}\right)=\frac{\bar{p}_{\imath} p_{j}+1}{p_{j}-p_{\imath}}=\frac{\infty p_{j}+1}{p_{j}-\infty}=-p_{\jmath}, \\
& F_{j}\left(p_{\imath}\right)=\frac{\bar{p}_{j} p_{\imath}+1}{p_{i}-p_{j}}=\frac{\bar{p}_{j} \infty+1}{\infty-p_{j}}=\bar{p}_{j}, \\
& \frac{b_{i}}{z-p_{\imath}}=\frac{b_{i}}{z-\infty}=0 .
\end{aligned}
$$

Proof of Corollary. Apply Theorem to the case when $a_{i} \equiv 1$ and $b_{\imath} \equiv$ $1 / \sqrt{n-1}$.

q.e.d.

Remark 2.1. By the proof of Theorem, we can observe the flux formula from another point of view. Namely we see that

$$
\begin{aligned}
& \sum_{i=1}^{n} a_{i} v_{i 1}=\sum_{i=1}^{n} \operatorname{Res}_{z=p_{\imath}}\left\{\frac{\left(1-g^{2}\right) f^{2}}{2}\right\}=-\sum_{\imath, \jmath=1 ; \imath \neq j}^{n} b_{\imath} b_{j} \frac{p_{\imath} p_{j}-1}{p_{i}-p_{\jmath}}=0, \\
& \sum_{\imath=1}^{n} a_{i} v_{\imath 2}=\sum_{i=1}^{n} \operatorname{Res}_{z=p_{\imath}}\left\{\frac{\sqrt{-1}\left(1+g^{2}\right) f^{2}}{2}\right\}=-\frac{1}{\sqrt{-1}} \sum_{\imath, j=1 ; i \neq j}^{n} b_{i} b_{j} \frac{p_{\imath} p_{j}+1}{p_{i}-p_{\jmath}}=0, \\
& \sum_{\imath=1}^{n} a_{i} v_{i 3}=\sum_{i=1}^{n} \operatorname{Res}_{z=p_{i}}\left\{g f^{2}\right\}=-\sum_{\imath, \jmath=1 ; i \neq j}^{n} b_{i} b_{j} \frac{p_{i}+p_{\jmath}}{p_{i}-p_{\jmath}}=0 .
\end{aligned}
$$




\section{Examples}

First, we remark that the linear transformation $F_{i}(z)$ defined in Section 1 is identified with an isometry of the unit sphere $\boldsymbol{S}^{2}=\sigma^{-1}(\widehat{\boldsymbol{C}})$ such that $F_{i}\left(p_{2}\right)=\infty$ and $F_{i}\left(-1 / \bar{p}_{\imath}\right)=0$. Therefore, if the subset $\left\{v_{j}\right\}_{j=1}^{k}$ of $\boldsymbol{S}^{2}-\left\{\boldsymbol{\sigma}^{-1}\left(p_{\imath}\right)\right\}$ is invariant under the action of some nontrivial subgroup of $S O(3)$ which fixes $\sigma^{-1}\left(p_{2}\right)$, then clearly $\sum_{j=1}^{k} F_{i}\left(\sigma\left(v_{j}\right)\right)=0$ (cf. Xu [9, Lemma 4.6]). By this observation, we get the following example without any more computation.

Example 3.1. (Families of polyhedrally symmetric minimal surfaces) Let $P$ be a regular polyhedron inscribed to the unit sphere $\boldsymbol{S}^{2}$ in $\boldsymbol{R}^{3},\left\{v_{j}\right\}_{j=1}^{k}$ the set of the vertices of $P,\left\{v_{j}^{\prime}\right\}_{j=1}^{k^{\prime}}$ the set of the centers of the edges of $P$, and $\left\{v_{j}^{\prime \prime}\right\}_{j=1}^{k^{\prime \prime}}$ the set of the varycenters of the faces of $P$. It is well-known that

$$
\left(k, k^{\prime}, k^{\prime \prime}\right)= \begin{cases}(4,6,4) & \text { if } P \text { is a regular tetrahedron, } \\ (8,12,6) & \text { if } P \text { is a cube, } \\ (6,12,8) & \text { if } P \text { is a regular octahedron, } \\ (20,30,12) & \text { if } P \text { is a regular dodecahedron, } \\ (12,30,20) & \text { if } P \text { is a regular icosahedron. }\end{cases}
$$

Set $p_{\jmath}:=\sigma\left(v_{j}\right), p_{j}^{\prime}:=\sigma\left(v_{j}^{\prime} /\left|v_{j}^{\prime}\right|\right)$, and $p_{\jmath}^{\prime \prime}:=\sigma\left(v_{\jmath}^{\prime \prime} /\left|v_{\jmath}^{\prime \prime}\right|\right)$. For any real numbers $b$, $b^{\prime}$ and $b^{\prime \prime}$, define a surface $X_{\left(b, b^{\prime}, b^{\prime \prime}\right)}: \hat{\boldsymbol{C}}-\left\{p_{1}, \cdots, p_{k}, p_{1}^{\prime}, \cdots, p_{k^{\prime}}^{\prime}, p_{1}^{\prime \prime}, \cdots, p_{k^{\prime \prime}}^{\prime \prime}\right\} \rightarrow \boldsymbol{R}^{3}$ by the data

$$
f(z):=b \sum_{j=1}^{k} \frac{1}{z-p_{\jmath}}+b^{\prime} \sum_{j=1}^{k^{\prime}} \frac{1}{z-p_{\jmath}^{\prime}}+b^{\prime \prime} \sum_{j=1}^{k^{\prime \prime}} \frac{1}{z-p_{j}^{\prime \prime}}, \quad \beta:=k b+k^{\prime} b^{\prime}+k^{\prime \prime} b^{\prime \prime} .
$$

Then $\left\{X_{\left(b, b^{\prime}, b^{\prime \prime}\right)}\right\}$ is a 3 -parameter family of minimal surfaces which are invariant under the action of the polyhedral group $\Gamma_{P}$ corresponding to $P$. For a generic $\left(b, b^{\prime}, b^{\prime \prime}\right), X_{\left(b, b^{\prime}, b^{\prime \prime}\right)}$ has $k+k^{\prime}+k^{\prime \prime}$ catenoid ends whose weights take 3 different values. More precisely, by using Lemma A.1 in Appendix, we see that, for any positive numbers $a, a^{\prime}$ and $a^{\prime \prime}$, there exists $a \Gamma_{p}$-invariant $\left(k+k^{\prime}+k^{\prime \prime}\right)$ end catenoid $X_{\left(b, b^{\prime}, b^{\prime \prime}\right)}$ such that

$$
\begin{aligned}
& g\left(p_{j}\right)=p_{\jmath}, \quad w\left(p_{\jmath}\right)=a \quad j=1, \cdots, k, \\
& g\left(p_{j}^{\prime}\right)=p_{\jmath}^{\prime}, \quad w\left(p_{j}^{\prime}\right)=a^{\prime} \quad \jmath=1, \cdots, k^{\prime}, \\
& g\left(p_{\jmath}^{\prime \prime}\right)=p_{\jmath}^{\prime \prime}, \quad w\left(p_{\jmath}^{\prime \prime}\right)=a^{\prime \prime} \quad j=1, \cdots, k^{\prime \prime} .
\end{aligned}
$$

When one of $b, b^{\prime}$ and $b^{\prime \prime}$ vanishes, since $k, k^{\prime}$ or $k^{\prime \prime}$ ends are removed, it has $k^{\prime}+k^{\prime \prime}, k+k^{\prime \prime}$ or $k+k^{\prime}$ ends. By using Lemma A.2, we see that, for any

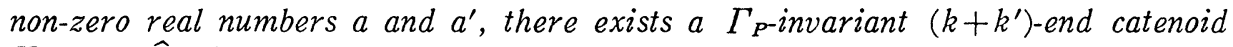
$X_{\left(b, b^{\prime}, 0\right)}: \widehat{\boldsymbol{C}}-\left\{p_{1}, \cdots, p_{k}, p_{1}^{\prime}, \cdots, p_{k^{\prime}}^{\prime}\right\} \rightarrow \boldsymbol{R}^{3}$ satisfying the conditions (3.1) and (3.2). Indeed, in the construction above, we may choose purely imaginary numbers $b$, 
$b^{\prime}$ and $b^{\prime \prime}$ in place of real numbers, and, by Lemma A.2, we get $X_{\left(b, b^{\prime}, 0\right)}$ as above. Of course, the same assertion holds also in the case with $b^{\prime}=0$ or $b=0$.

$\mathrm{Xu}$ [9], Rossman [7] and Umehara-Yamada [8] studied the special cases of this type when two of $b, b^{\prime}$ and $b^{\prime \prime}$ vanish.

The dihedral version of this type is the following

Example 3.2. (Families of $D_{k}$-invariant minimal surfaces) Let $k$ be an integer greater than 1 . For any real numbers $b, b^{\prime}$ and $b^{\prime \prime}$, define a surface $X_{\left(b, b^{\prime}, b^{\prime \prime}\right)}: \widehat{\boldsymbol{C}}-\left\{1, \zeta_{2 k}, \cdots, \zeta_{2 k}^{2 k-1}, \infty, 0\right\} \rightarrow \boldsymbol{R}^{3}$ by the data

$$
f(z):=b \frac{k z^{k-1}}{z^{k}-1}+b^{\prime} \frac{k z^{k-1}}{z^{k}+1}+\frac{b^{\prime \prime}}{z}, \quad \beta:=k\left(b+b^{\prime}\right)+2 b^{\prime \prime} .
$$

Then $\left\{X_{\left(b, b^{\prime}, b^{\prime \prime}\right)}\right\}$ is a 3-parameter family of $D_{k}$-invariant minimal surfaces. For a generic $\left(b, b^{\prime}, b^{\prime \prime}\right), X_{\left(b, b^{\prime}, b^{\prime \prime}\right)}$ has $2 k+2$ catenoid ends whose weights take 3 different values. More precisely, by using Lemma A.1, we see that, for any positive numbers $a, a^{\prime}$ and $a^{\prime \prime}$, there exists a $D_{k}$-invariant $(2 k+2)$-end catenond $X_{\left(b, b^{\prime}, b^{\prime \prime}\right)}$ such that

$$
\begin{aligned}
& g\left(\zeta_{k}^{j}\right)=\zeta_{k}^{j}, \quad w\left(\zeta_{k}^{j}\right)=a \quad j=0, \cdots, k-1, \\
& g\left(\zeta_{2 k}^{2 J-1}\right)=\zeta_{2 k}^{2 J-1}, \quad w\left(\zeta_{2 k}^{2 J-1}\right)=a^{\prime} \quad \jmath=1, \cdots, k, \\
& g(\infty)=\infty, \quad g(0)=0, \quad w(\infty)=w(0)=a^{\prime \prime} .
\end{aligned}
$$

When $b^{\prime \prime}\left(\right.$ resp. $\left.b^{\prime}\right)=0$, it has $2 k$ (resp. $k+2$ ) ends and the similar result as above also holds. It was partially obtained by Karcher [4] $(k=2), \mathrm{Xu}[9]$ and Rossman [7] $(k \geqq 3)$. More generally, by Lemma A.2 and the same consideration as in Example 3.1, we see that, for any non-zero real numbers $a$ and $a^{\prime}$, there exists a $D_{k}$-invariant $2 k$-end catenoid $X_{\left(b, b^{\prime}, 0\right)}: \widehat{\boldsymbol{C}}-\left\{1, \boldsymbol{\zeta}_{2 k}, \cdots, \boldsymbol{\zeta}_{2 k}^{2 k-1}\right\} \rightarrow \boldsymbol{R}^{3}$ satisfying the conditions (3.4) and (3.5), and that, for any non-zero real numbers $a$ and $a^{\prime \prime}$, there exists $a D_{k}$-invariant $(k+2)$-end catenoid $X_{\left(b, 0, b^{\prime \prime}\right)}: \widehat{\boldsymbol{C}}-\left\{1, \zeta_{k}, \cdots\right.$, $\left.\zeta_{k}^{k-1}, \infty, 0\right\} \rightarrow \boldsymbol{R}^{3}$ satısfying the conditions (3.4) and (3.6).

When $b^{\prime}=b^{\prime \prime}=0$, we get the examples in Jorge-Meeks [3].

By the consideration in Examples 3.1-2, we can observe that there are essentially different $n$-end catenoids with the same data $v_{1}, \cdots, v_{n}, a_{1}, \cdots, a_{n}$. For example, in Example 3.2, applying Lemma A.2 for $\left(k, k^{\prime}\right)=(k, 2)$, we see that, for any non-zero real numbers $a$ and $a^{\prime \prime}$ such that $k^{2} a \neq 4 a^{\prime \prime}$, there exist two $D_{k}$-invariant $(k+2)$-end catenoids $X_{\left(b_{ \pm}, 0, b_{ \pm}^{\prime \prime}\right)}$ satisfying the conditions (3.4) and (3.6). Since the metric of $X_{\left(b, 0, b^{\prime \prime}\right)}$ is given by

$$
\frac{\left[\left|\left(k b+b^{\prime \prime}\right) z^{k}-b^{\prime \prime}\right|^{2}+\left|z\left\{\left(k b+b^{\prime \prime}\right)-b^{\prime \prime} z^{k}\right\}\right|^{2}\right]^{2}}{\left|z\left(z^{k}-1\right)\right|^{4}}|d z|^{2}
$$

and $\left|b_{+}^{\prime \prime}\right| \neq\left|b_{-}^{\prime \prime}\right|$ if $k^{2} a \neq 2 a^{\prime \prime}, X_{\left(b_{+}, 0, b_{+}^{\prime \prime}\right)}$ and $X_{\left(b_{-}, 0, b_{-}^{\prime \prime}\right)}$ are not isometric with each 
other for generic $a$ and $a^{\prime \prime}$.

We can deform the surfaces in Example 3.2 to the following

Example 3.3. (Families of $C_{k}$-invariant minimal surfaces) Let $k$ be an integer greater than 1. For any non-zero real number $p$ and real numbers $b$ and $b^{\prime}$, define the surface $X_{\left(p, b, b^{\prime}\right)}: \widehat{\boldsymbol{C}}-\left\{p, p \boldsymbol{\zeta}_{k}, \cdots, p \boldsymbol{\zeta}_{k}^{k-1}, \infty, 0\right\} \rightarrow \boldsymbol{R}^{3}$ by the data

$$
f(z):=b \frac{k z^{k-1}}{z^{k}-p^{k}}+\frac{b^{\prime \prime}}{z}, \quad \beta:=k b+b^{\prime}+b^{\prime \prime},
$$

where $b^{\prime \prime}:=(k-1)\left(p^{2}-1\right) b / 2+p^{2} b^{\prime}$. Then $\left\{X_{\left(p, b, b^{\prime}\right)}\right\}$ is a 3-parameter family of $C_{k}$-invariant minimal surfaces.

Indeed it is clear that the condition (1.2) holds with $p_{\imath}=\infty$ and 0 , and we have only to check it with $p_{i}=p \zeta_{k}^{l}(l=0,1, \cdots, k-1)$. By direct computation,

$$
\begin{aligned}
b \sum_{j=1}^{k-1} \frac{p \zeta_{k}^{-l} p \zeta_{k}^{l+j}+1}{p \zeta_{k}^{l+}-p \zeta_{k}^{l}}+b^{\prime} \frac{p \zeta_{k}^{-l} \infty+1}{\infty-p \zeta_{k}^{l}}+b^{\prime \prime} \frac{p \zeta_{k}^{-l} 0+1}{0-p \zeta_{k}^{l}} \\
\quad=b \sum_{j=1}^{k-1} \frac{p^{2} \zeta_{k}^{2}+1}{p \zeta_{k}^{l}\left(\zeta_{k}^{j}-1\right)}+b^{\prime} \frac{p}{\zeta_{k}^{l}}-b^{\prime \prime} \frac{1}{p \zeta_{k}^{l}} \\
\quad=\frac{1}{p \zeta_{k}^{l}}\left\{b \sum_{j=1}^{k-1} \frac{p^{2} \zeta_{k}^{j}+1}{\zeta_{k}^{j}-1}+b^{\prime} p^{2}-b^{\prime \prime}\right\} \\
\quad=\frac{1}{p \zeta_{k}^{l}}\left\{\frac{(k-1)\left(p^{2}-1\right) b}{2}+p^{2} b^{\prime}-b^{\prime \prime}\right\} \\
=0 .
\end{aligned}
$$

Hence the surface $X_{\left(p, b, b^{\prime}\right)}$ is well-defined.

For a generic $\left(p, b, b^{\prime}\right), X_{\left(p, b, b^{\prime}\right)}$ has $k+2$ catenoid ends whose weights take 3 different values. More precisely, by using Lemma A.3, we see that, for any non-zero real number $p$ and positive numbers $a, a^{\prime}$ and $a^{\prime \prime}$ satisfying $a \sum_{j=0}^{k-1} \sigma^{-1}\left(p \zeta_{k}^{j}\right)$ $+a^{\prime} \sigma^{-1}(\infty)+a^{\prime \prime} \sigma^{-1}(0)=0$ (i.e. $\left.a k\left(p^{2}-1\right) /\left(p^{2}+1\right)+a^{\prime}-a^{\prime \prime}=0\right)$, there exists a $C_{k^{-}}$ invariant $(k+2)$-end catenoid $X_{\left(p, b, b^{\prime}\right)}$ such that

$$
\begin{aligned}
g\left(p \zeta_{k}^{\jmath}\right) & =\zeta_{k}^{\jmath}, & & w\left(p \zeta_{k}^{\jmath}\right)=a \quad j=0, \cdots, k-1, \\
g(\infty) & =\infty, & & w(\infty)=a^{\prime}, \\
g(0) & =0, & & w(0)=a^{\prime \prime} .
\end{aligned}
$$

Karcher [4] constructed this example with $k=2$.

When $b^{\prime}=0$, since the end $\infty$ is removed, it has $k+1$ ends. $\mathrm{Xu}$ [9] constructed this example with $\left(p, b^{\prime}\right)=(\sqrt{(k+1) /(k-1)}, 0)$. However, more generally, by using Lemma A.2, we see that, for any non-zero real number $p \neq \pm 1$ and non-zero real numbers $a$ and $a^{\prime \prime}$ satisfying $a \sum_{j=0}^{k-1} \sigma^{-1}\left(p \zeta_{k}^{j}\right)+a^{\prime \prime} \sigma^{-1}(0)=0$ (i.e. $\left.a k\left(p^{2}-1\right) /\left(p^{2}+1\right)-a^{\prime \prime}=0\right)$, there exists a $C_{k}$-invariant $(k+1)$-end catenoid $X_{(p, b, 0)}$ : $\hat{\boldsymbol{C}}-\left\{p, p \boldsymbol{\zeta}_{k}, \cdots, p \zeta_{k}^{k-1}, 0\right\} \rightarrow \boldsymbol{R}^{3}$ satisfying the conditions (3.7) and (3.9). 
See Figure 3.1 for the arrangements of the ends. We point to the positions of the ends by the symbols $\bigcirc, \bullet$ and $\triangle$. Any two ends of different symbols may have weights different from each other.

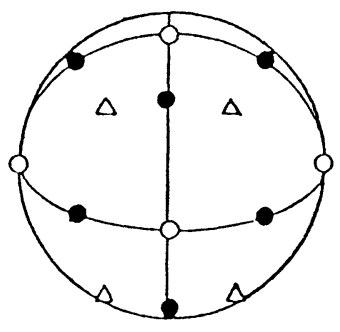

Example 3.1

$P$ is a regular octahedron

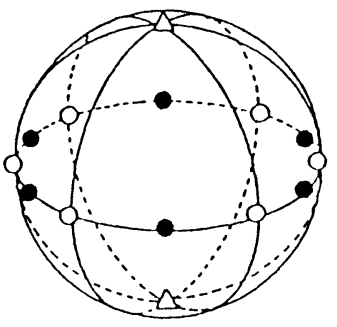

Example 3.2 $k=6$

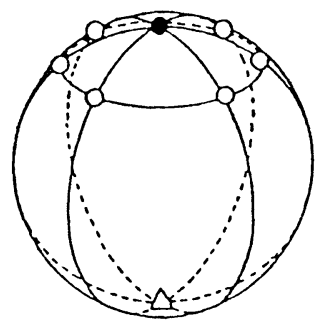

Example 3.3 $k=6$

Figure 3.1.

By the similar computation as in Example 3.3, we get various examples of families of $n$-end catenoids, e.g. as follows.

Example 3.4. (Example 3.2 plus $2 k$ ends) Let $k$ be an integer greater than 1. For any non-zero complex number $\xi$ such that $\xi^{2 k} \neq 1$, and any real numbers $b, b^{\prime}, b^{\prime \prime}$ and $b^{\prime \prime \prime}$ satisfying

$$
\begin{aligned}
2 k & \left\{\left(\xi^{k}+|\xi|^{2}\right)\left(\xi^{k}+1\right) b+\left(\xi^{k}-|\xi|^{2}\right)\left(\xi^{k}-1\right) b^{\prime}\right\} \\
& =2\left(|\xi|^{2}-1\right)\left(\xi^{2 k}-1\right) b^{\prime \prime}+\left\{(k-1)\left(|\xi|^{2} \xi^{2 k}+1\right)-(3 k-1)\left(\xi^{2 k}+|\xi|^{2}\right)\right\} b^{\prime \prime \prime},
\end{aligned}
$$

define a surface $X_{\left(\xi, b, b^{\prime}, b^{\prime \prime}, b^{\prime \prime \prime}\right)}: \hat{\boldsymbol{C}}-\left\{1, \zeta_{2 k}, \cdots, \zeta_{2 k}^{2 k-1}, \infty, 0, \xi^{ \pm 1}, \xi^{ \pm 1} \zeta_{k}, \cdots, \xi^{ \pm 1} \zeta_{k}^{k-1}\right\} \rightarrow \boldsymbol{R}^{3}$ by the data

$$
f(z):=b \frac{k z^{k-1}}{z^{k}-1}+b^{\prime} \frac{k z^{k-1}}{z^{k}+1}+\frac{b^{\prime \prime}}{z}+b^{\prime \prime \prime}\left(\frac{k z^{k-1}}{z^{k}-\xi^{k}}+\frac{k z^{k-1}}{z^{k}-\xi^{-k}}\right), \quad \beta:=k\left(b+b^{\prime}+2 b^{\prime \prime \prime}\right)+2 b^{\prime \prime} .
$$

Since we assume (3.10), the condition (1.2) in Theorem is satisfied and $\left\{X_{\left(\xi, b, b^{\prime}, b^{\prime \prime}, b^{\prime \prime \prime}\right)}\right\}$ is a family of $D_{k}$-invariant minimal surfaces. For a generic $\left(\xi, b, b^{\prime}, b^{\prime \prime}, b^{\prime \prime \prime}\right)$ satisfying the condition (3.10), $X_{\left(\xi, b, b^{\prime}, b^{\prime \prime}, b^{\prime \prime \prime}\right)}$ has $4 k+2$ catenoid ends whose weights take 4 different values. However, when some of $b, b^{\prime}$ and $b^{\prime \prime}$ vanish, it has $4 k, 3 k+2,3 k, 2 k+2$ or $2 k$ ends.

Here we claim that, for any $\xi$, there are infinitely many $\left(b, b^{\prime}, b^{\prime \prime}, b^{\prime \prime \prime}\right) \in \boldsymbol{R}^{4}$ satisfying the condition (3.10).

CASE 1. When $\xi^{k} \in \boldsymbol{R}$ i.e. $\xi=p \zeta_{2 k}^{\jmath}$ for some non-zero real number $p(\neq \pm 1)$ and some integer $j$, the condition (3.10) is satisfied if and only if the following condition holds :

$$
\begin{aligned}
2 k p^{2} & {\left[\left\{p^{k-2}+(-1)^{j}\right\}\left\{p^{k}+(-1)^{j}\right\} b+\left\{p^{k-2}-(-1)^{j}\right\}\left\{p^{k}-(-1)^{j}\right\} b^{\prime}\right] } \\
& =2\left(p^{2}-1\right)\left(p^{2 k}-1\right) b^{\prime \prime}+\left\{(k-1)\left(p^{2 k+2}+1\right)-(3 k-1) p^{2}\left(p^{2 k-2}+1\right)\right\} b^{\prime \prime \prime} .
\end{aligned}
$$


Hence the claim above is justified. In this case, $X_{\left(\xi, b, b^{\prime}, b^{\prime \prime}, b^{\prime \prime}\right)}$ has the symmetry of a regular $k$-angular prism.

CASE 2. If we set $p:=|\xi|$ and $r:=\operatorname{Re} \xi^{k} /|\xi|^{k}$, then, by using the equality $\xi^{2 k}=2 r p^{k} \xi^{k}-p^{2 k}$, we can rewrite the condition (3.10) as follows.

$$
\begin{aligned}
& 2 k\left[\left\{\left(2 r p^{k}+p^{2}+1\right) \xi^{k}-p^{2}\left(p^{2 k-2}-1\right)\right\} b+\left\{\left(2 r p^{k}-p^{2}-1\right) \xi^{k}-p^{2}\left(p^{2 k-2}-1\right)\right\} b^{\prime}\right] \\
&= 2\left(p^{2}-1\right)\left\{2 r p^{k} \xi^{k}-\left(p^{2 k}+1\right)\right\} b^{\prime \prime} \\
&+\left[(k-1)\left\{2 r p^{k+2} \xi^{k}-\left(p^{2 k+2}-1\right)\right\}-(3 k-1)\left\{2 r p^{k} \xi^{k}-p^{2}\left(p^{2 k-2}-1\right)\right\}\right] b^{\prime \prime \prime} .
\end{aligned}
$$

Therefore, when $\xi^{k} \notin \boldsymbol{R}$, the condition (3.10) is satisfied if and only if both of the following conditions hold:

$$
\begin{aligned}
& 2 k p^{2}\left(p^{2 k-2}-1\right)\left(b+b^{\prime}\right) \\
& \quad=2\left(p^{2}-1\right)\left(p^{2 k}+1\right) b^{\prime \prime}+\left\{(k-1)\left(p^{2 k+2}-1\right)-(3 k-1) p^{2}\left(p^{2 k-2}-1\right)\right\} b^{\prime \prime} ; \\
& k\left\{\left(2 r p^{k}+p^{2}+1\right) b+\left(2 r p^{k}-p^{2}-1\right) b^{\prime}\right\} \\
& \quad=r p^{k}\left[2\left(p^{2}-1\right) b^{\prime \prime}+\left\{(k-1) p^{2}-(3 k-1)\right\} b^{\prime \prime \prime}\right] .
\end{aligned}
$$

Hence, also in this case, the claim above is justified. Let us observe this case more concretely.

(1) When $\xi^{k} \in \sqrt{-1} \boldsymbol{R}$ i.e. $\xi=p \zeta_{4 k}^{2 J-1}$ for some non-zero real number $p$ and some integer $j$, the condition (3.10) is satisfied if and only if both of the following conditions hold:

$$
\begin{aligned}
& 4 k p^{2}\left(p^{2 k-2}-1\right) b \\
& \quad=2\left(p^{2}-1\right)\left(p^{2 k}+1\right) b^{\prime \prime}+\left\{(k-1)\left(p^{2 k+2}-1\right)-(3 k-1) p^{2}\left(p^{2 k-2}-1\right)\right\} b^{\prime \prime \prime} ; \\
& b=b^{\prime} .
\end{aligned}
$$

In this case, $X_{\left(\xi, b, b^{\prime}, b^{\prime \prime}, b^{\prime \prime}\right)}$ has the symmetry of a regular $k$-angular antiprism.

(2) When $\xi^{2 k} \notin \boldsymbol{R}$ i. e. $\xi^{k} \notin \boldsymbol{R} \cup \sqrt{-1} \boldsymbol{R}$, the condition (3.10) is satisfied if and only if both of the following conditions hold:

$$
\begin{aligned}
& 2(k-1) r p^{k}\left(p^{2}-1\right)\left(b+b^{\prime}\right)+\left\{(k-1)\left(p^{2 k+2}-1\right)-(3 k-1) p^{2}\left(p^{2 k-2}-1\right)\right\}\left(b-b^{\prime}\right) \\
& \quad=4 r p^{k}\left(p^{2}-1\right) b^{\prime \prime} ; \\
& \left(2 r p^{k}+p^{2 k}+1\right) b+\left(2 r p^{k}-p^{2 k}-1\right) b^{\prime}+2 r p^{k} b^{\prime \prime \prime}=0 .
\end{aligned}
$$

In this case, if $|\xi| \neq 1$ and $b^{\prime \prime \prime} \neq 0$, then $X_{\left(\xi, b, b^{\prime}, b^{\prime \prime}, b^{\prime \prime \prime}\right)}$ has neither the symmetry of a regular $k$-angular prism nor the symmetry of a regular $k$-angular antiprism.

(3) When $|\xi|=1$, the condition (3.11) is automatically satisfied. Therefore the condition (3.10) is satisfied if and only if the following condition holds:

$$
(r+1) b+(r-1) b^{\prime}+r b^{\prime \prime \prime}=0 .
$$


In this case, we can choose $b^{\prime \prime}$ independently.

See Figure 3.2 for the arrangements of the additional ends $\xi^{ \pm 1} \zeta_{k}^{j}$ in each case above.

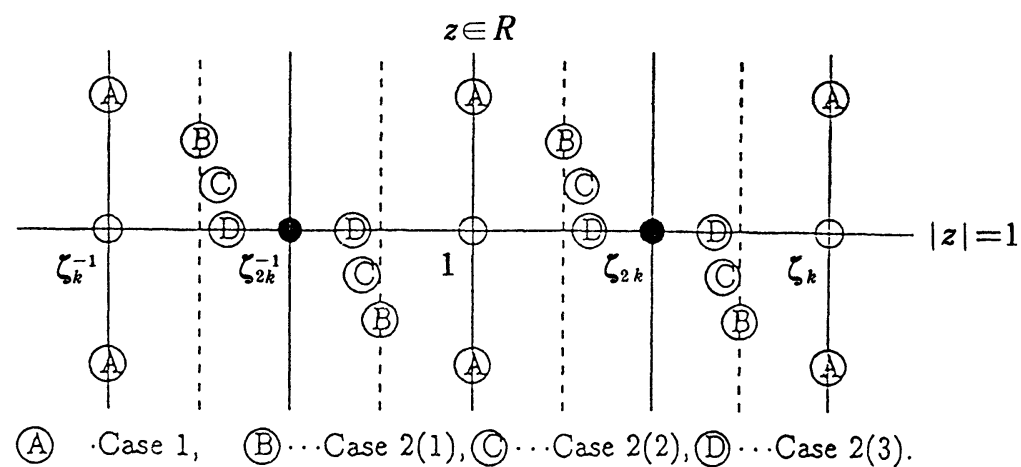

Figure 3.2.

Now we will describe a less symmetric

Example 3.5. (All of the 3-end catenoids) For any non-zero real numbers $p$ and $p^{\prime}$ such that $\left(p-p^{\prime}\right)\left(p p^{\prime}+1\right) \neq 0$, and any non-zero real or purely imaginary number $b$, define a surface $X_{\left(p, p^{\prime}, b\right)}: \hat{\boldsymbol{C}}-\left\{p, p^{\prime}, 0\right\} \rightarrow \boldsymbol{R}^{3}$ by the data

$$
f(z):=b\left\{\frac{p\left(p-p^{\prime}\right)}{z-p}+\frac{p^{\prime}\left(p^{\prime}-p\right)}{z-p^{\prime}}+\frac{p p^{\prime}\left(p p^{\prime}+1\right)}{z}\right\}, \quad \beta:=b\left(p^{2} p^{\prime 2}+p^{2}+p^{\prime 2}-p p^{\prime}\right) .
$$

This representation gives the affirmative answer to our problem in the case when $n=3$ and $v_{i}$ 's are different from each other. Conversely, all of the 3-end catenoids are described by this. Lopez [5] recently proved this result by somewhat different but essentially the same representation.

In the case $n \geqq 4$, our problem is still open.

\section{Appendix}

Here we prove three lemmas which were used in the previous section.

LEMMA A.1. Let $n$ be an integer greater than 1 , and $a_{1}, \cdots, a_{n}$ positive numbers. If there are two indices $i_{1}$ and $i_{2}$ such that $a_{\imath_{1}}=a_{\imath_{2}}=\max _{1 \leqq \imath \leqq n} a_{\imath}$, then there are positive numbers, $b_{1}, \cdots, b_{n}$ satisfying

$$
b_{i} \sum_{j \in N_{\imath}} b_{j}=a_{\imath} \quad \imath=1, \cdots, n .
$$

In particular, if $a_{i}=a$, for some $i$ and $\jmath$, then we can choose $b_{i}$ and $b_{3}$ as the same value. 
Proof. We may assume $i_{1}=1$ and $\imath_{2}=2$ without loss of generality. Set

$$
\varphi(t):=(n-2) t-\sum_{\imath=1}^{n} \sqrt{t^{2}-4 a_{\imath}} .
$$

It is clear that $\varphi(t)$ is a continuous function on $\left[2 \sqrt{a_{1}},+\infty\right)$ and $\lim _{t \cdot++\infty} \varphi(t)<0$. On the other hand, by the assumption, we have

$$
\begin{aligned}
\varphi\left(2 \sqrt{a_{1}}\right) & =2(n-2) \sqrt{a_{1}}-2 \sum_{i=1}^{n} \sqrt{a_{1}-a_{\imath}} \\
& =2 \sum_{i=3}^{n}\left(\sqrt{a_{1}}-\sqrt{a_{1}-a_{\imath}}\right) \\
& \geqq 0 .
\end{aligned}
$$

Hence, by the intermediate value theorem, there is a positive number $\tau \geqq 2 \sqrt{a_{1}}$ such that $\varphi(\tau)=0$. Set

$$
b_{i}:=\frac{\tau-\sqrt{\tau^{2}-4 a_{\imath}}}{2} \quad i=1, \cdots, n .
$$

Then, for any $i, b_{i}$ is positive and

$$
\begin{aligned}
b_{i} \sum_{j \in N_{\imath}} b_{j} & =\frac{\tau-\sqrt{\tau^{2}-4 a_{2}}}{2} \sum_{j \in N_{\imath}} \frac{\tau-\sqrt{\tau^{2}-4 a_{j}}}{2} \\
& =\frac{\tau-\sqrt{\tau^{2}-4 a_{\imath}}}{2} \times \frac{(n-1) \tau-\left\{(n-2) \tau-\sqrt{\tau^{2}-4 a_{i}}\right\}}{2} \\
& =a_{\imath},
\end{aligned}
$$

namely, $b_{1}, \cdots, b_{n}$ satisfy the condition (1.1).

q.e.d.

LEMMA A.2. Let $k$ be an integer greater than $1, k^{\prime}$ a positive integer, and $a$ and $a^{\prime}$ non-zero real numbers. Then there is at least one $\left(b, b^{\prime}\right)$ which belongs to either $\boldsymbol{R}^{2}$ or $(\sqrt{-1} \boldsymbol{R})^{2}$ and satisfies

$$
\left\{\begin{array}{l}
b\left\{(k-1) b+k^{\prime} b^{\prime}\right\}=a, \\
b^{\prime}\left\{k b+\left(k^{\prime}-1\right) b^{\prime}\right\}=a^{\prime}
\end{array}\right.
$$

and $k b+k^{\prime} b^{\prime} \neq 0$, if $k^{\prime}>1$, or if $k^{\prime}=1$ and $a^{\prime} \neq k a, k^{2} a$.

Proof. When $k^{\prime}>1$, solving the system of quadric equations (A.1), we get a solution $\left(b, b^{\prime}\right)=\left(b_{ \pm}, b_{ \pm}^{\prime}\right)$, where

and

$$
b_{ \pm}:=\sqrt{\frac{k^{2} a^{2}-k^{\prime 2} a^{\prime 2}-D \pm 2 k^{\prime} a^{\prime} \sqrt{D}}{4(k-1)\left(k+k^{\prime}-1\right) a^{\prime}}}, \quad b_{ \pm}^{\prime}:=\frac{a-(k-1) b_{ \pm}^{2}}{k^{\prime} b_{ \pm}}
$$

$$
D:=k^{2} a^{2}+2\left(k k^{\prime}-2 k-2 k^{\prime}+2\right) a a^{\prime}+k^{\prime 2} a^{\prime 2} .
$$


By the assumption and direct computation, we have $D>0$ and $k^{2} a^{2}-k^{\prime 2} a^{\prime 2}-D$ $\pm 2 k^{\prime} a^{\prime} \sqrt{D} \neq 0$. Therefore, we can easily see that $\left(b_{ \pm}, b_{ \pm}^{\prime}\right)$ belongs to either $\boldsymbol{R}^{2}$ or $(\sqrt{-1} \boldsymbol{R})^{2}$. Moreover, since

$$
k b_{ \pm}+k^{\prime} b_{ \pm}^{\prime}=\frac{a+b_{ \pm}^{2}}{b_{ \pm}}=\frac{a^{\prime}+b_{ \pm}^{\prime 2}}{b_{ \pm}^{\prime}},
$$

we have $k b_{+}+k^{\prime} b_{+}^{\prime} \neq 0$ or $k b_{-}+k^{\prime} b_{-.}^{\prime} \neq 0$. Indeed, if $k^{2} a \neq k^{\prime 2} a^{\prime}$ (resp. $k^{2} a=k^{\prime 2} a^{\prime}$ ), then each (resp. one) of $\left(b_{ \pm}, b_{ \pm}^{\prime}\right)$ satisfies $k b_{ \pm}+k^{\prime} b_{ \pm}^{\prime} \neq 0$.

On the other hand, when $k^{\prime}=1$ and $a^{\prime} \neq k a, k^{2} a$, solving the system of quadric equations (A.1), we get a solution

$$
\left(b, b^{\prime}\right):=\left(\sqrt{\frac{k a-a^{\prime}}{k(k-1)}}, \frac{a^{\prime}}{k b}\right) .
$$

We can easily see that $\left(b, b^{\prime}\right)$ belongs to either $\boldsymbol{R}^{2}$ or $(\sqrt{-1} \boldsymbol{R})^{2}$. Moreover, we have

$$
k b+b^{\prime}=\frac{k^{2} a-a^{\prime}}{k(k-1) b} \neq 0 .
$$

q. e. d,

LEMMA A.3. Let $k$ be an integer greater than 1 , and $a, a^{\prime}$ and $a^{\prime \prime}$ positive numbers. If $\max \left\{a, a^{\prime \prime}\right\} \leqq a^{\prime}<k a+a^{\prime \prime}$ or $\max \left\{a, a^{\prime}\right\} \leqq a^{\prime \prime}<k a+a^{\prime}$, then there are positive numbers $b, b^{\prime}$ and $b^{\prime \prime}$ satisfying

$$
\left\{\begin{array}{l}
b\left\{(k-1) b+b^{\prime}+b^{\prime \prime}\right\}=a, \\
b^{\prime}\left(k b+b^{\prime \prime}\right)=a^{\prime}, \\
b^{\prime \prime}\left(k b+b^{\prime}\right)=a^{\prime \prime} .
\end{array}\right.
$$

Proof. Solving the system of quadric equations (A.2), we get a solution $\left(b, b^{\prime}, b^{\prime \prime}\right)$, where

$$
\begin{aligned}
b & :=\sqrt{\frac{\sqrt{D-k\left(a^{\prime}+a^{\prime \prime}-a\right)}}{k\left(k^{2}-1\right)}}, \\
b^{\prime} & :=\frac{k^{2} a+(2 k+1) a^{\prime}-a^{\prime \prime}-\sqrt{D}}{2 k(k+1) b} \\
b^{\prime \prime} & :=\frac{k^{2} a+(2 k+1) a^{\prime \prime}-a^{\prime}-\sqrt{D}}{2 k(k+1) b}
\end{aligned}
$$

and

$$
D:=k^{4} a^{2}+a^{\prime 2}+a^{\prime \prime 2}-2 k^{2}\left(a^{\prime}+a^{\prime \prime}\right) a+2\left(2 k^{2}-1\right) a^{\prime} a^{\prime \prime} .
$$

By the assumption and direct computation, we have $D>0$ and

$$
0<k\left(a^{\prime}+a^{\prime \prime}-a\right)<\sqrt{D}<\min \left\{k^{2} a+(2 k+1) a^{\prime}-a^{\prime \prime}, k^{2} a+(2 k+1) a^{\prime \prime}-a^{\prime}\right\} .
$$

Therefore, we can easily see that $b, b^{\prime}$ and $b^{\prime \prime}$ are positive numbers. q.e.d. 


\section{REFERENCES}

[1] J. Berglund and W. Rossman, Minimal surfaces with catenoid ends, to appear in Pacific J. Math..

[2] D. Hoffman and W. H. Meeks III, Minimal surfaces based on the catenoid, Amer. Math. Monthly, 97 (1990), 702-730.

[3] L.P. JoRge AND W.H. MEeKs III, The topology of complete minimal surfaces of finite total Gaussian curvature, Topology, 22 (1983), 203-221.

[4] H. Karcher, Construction of minimal surfaces, Surveys in Geometry 1989/1990, University of Tokyo.

[5] F. J. LOPEZ, The classification of complete minimal surfaces with total curvature greater than $-12 \pi$, Trans. Amer. Math. Soc., 334 (1992), 49-74.

[6] R. Osserman, A survey of minimal surfaces, Van Nostrand, New York, 1969.

[7] W. Rossman, Minimal surfaces in $\boldsymbol{R}^{3}$ with the symmetry group $D_{n} \times \boldsymbol{Z}_{2}$, to appear in Tohoku Math. J..

[8] M. UMEHARA AND K. YAmAda, Surfaces of constant mean curvature $c$ in $\boldsymbol{H}^{3}\left(-c^{2}\right)$ with prescribed hyperbolic Gauss map, preprint.

[9] Y. XU, Symmetric minimal surfaces in $\boldsymbol{R}^{3}$, to appear in Pacific J. Math..

Department of Mathematics

NARA WOMEN'S UNIVERSITY

NARA 630, JAPAN

Present Address :

Department of Mathematics

Osaka UnIVERsity

TOYONAKA, OsAKa 560, JAPAN 